\title{
Power-level regulation and simulation of nonlinear pressurized water reactor core with xenon oscillation using $\mathrm{H}$-infinity loop shaping control
}

\author{
Gang $\mathrm{Li}^{1,2}$, Bin Liang ${ }^{1,2}$, a Xueqian Wang ${ }^{1,2}, \mathrm{Xiu} \mathrm{Li}^{1,2}$ and Kang Wang ${ }^{1,2}$ \\ ${ }^{1}$ Tsinghua University, National Laboratory for Information Science and Technology, 100084 Beijing, China \\ ${ }^{2}$ Shenzhen Key Lab of Space Robotic Technology and Telescience, 518055 Shenzhen, China
}

\begin{abstract}
This investigation is to solve the power-level control issue of a nonlinear pressurized water reactor core with xenon oscillations. A nonlinear pressurized water reactor core is modeled using the lumped parameter method, and a linear model of the core is then obtained through the small perturbation linearization way. The Holoop shapingcontrolis utilized to design a robust controller of the linearized core model.The calculated Holoop shaping controller is applied to the nonlinear core model. The nonlinear core model and the Ho loop shaping controller build the nonlinear core power-level Hœloop shaping control system.Finally, the nonlinear core power-level Hœloop shaping control system is simulatedconsidering two typical load processes that are a step load maneuver and a ramp load maneuver, and simulation results show that the nonlinear control system is effective.
\end{abstract}

\section{Introduction}

Nuclear energy as a clean energy can be sustainable and renewable. Hence, developing technologies of nuclear power plants (NPPs) for electricity generation is inevitable. Of these technologies, it is necessary to investigate the power control technology of nonlinear nuclear reactor cores with xenon oscillations.

Several researchers have made great efforts for the improvement of reactor core power control systems. The state-feedback basedcontrol with classical control part was proposed by Edwards et al. [1-2] to devise power control systems of pressurized water reactor (PWR) cores. The linear quadratic Gaussian with loop transfer recovery (LQG/LTR) control wasadopted to contrivePWR core controllers for power regulations [3-4]. However, the controllers in references [1-4] are designed based on a linear core model without xenon oscillations, and not always optimal or even ineffective for nonlinear cores.

Based on this consideration, the Holoop shaping control [5] with stronger robustness than that of the statefeedback or LQG/LTR control is adopted to design a nonlinear power control system ofa PWR core with xenon oscillations. Finally, the nonlinear PWR core power Hooloop shaping control system is simulated and conclusions are drawn.

\section{Modeling for PWR core}

The According to the point reactor core modeling [6-8], the nonlinear PWR core is modeled adopting the point kinetics equations with six groups of delayed neutrons and reactivity feedbacks due to control rod movement

\footnotetext{
a Corresponding author: wychug@163.com
}

and variations in fuel temperature, coolant temperature and xenon concentration. Main model parameters are given in Table 1.The nonlinear core model is showed as Eq.(1).

$$
\left\{\begin{array}{l}
\frac{d P_{r}}{d t}=\frac{\rho-\beta}{\Lambda} P_{r}+\sum_{i=1}^{g} \frac{\beta_{i} c_{r i}}{\Lambda} \\
\frac{d c_{r i}}{d t}=\lambda_{i} P_{r}-\lambda_{i} c_{r i}, i=1,2, \ldots, g \\
\frac{d I_{r}}{d t}=\frac{\gamma_{I} P_{f 0}}{E_{e f f} V I_{f 0}} P_{r}-\lambda_{I} I_{r} \\
\frac{d X_{r}}{d t}=\frac{\gamma_{X} P_{f 0}}{E_{e f f} V X_{f 0}} P_{r}+\frac{\lambda_{I} I_{f 0}}{X_{f 0}} I_{r}-\left(\lambda_{X}+\frac{\sigma_{X} P_{f 0}}{E_{e f f} \Sigma_{f} V} P_{r}\right) X_{r} \\
\frac{d T_{f}}{d t}=\frac{f_{f} P_{0}}{\mu_{f}} P_{r}-\frac{\Omega}{\mu_{f}} T_{f}+\frac{\Omega}{2 \mu_{f}} T_{i}+\frac{\Omega}{2 \mu_{f}} T_{e} \\
\frac{d T_{e}}{d t}=\frac{\left(1-f_{f}\right) P_{0}}{\mu_{c}} P_{r}+\frac{\Omega}{\mu_{c}} T_{f}+\frac{2 M-\Omega}{2 \mu_{c}} T_{i}-\frac{2 M+\Omega}{2 \mu_{c}} T_{e} \\
\rho=\rho_{r o d}+\alpha_{f}\left(T_{f}-T_{f 0}\right)+\frac{\alpha_{c}}{2}\left(T_{i}-T_{i 0}\right)+\frac{\alpha_{c}}{2}\left(T_{e}-T_{e 0}\right) \\
\quad+\alpha_{x} X_{f 0}\left(X_{r}-X_{r 0}\right) \\
\delta \rho_{r o d}=\alpha_{r o d} \delta r o d
\end{array}\right.
$$


Table 1. Main model parameters.

\begin{tabular}{|c|c|}
\hline Parameter & Name \\
\hline$P_{r}$ & core power level \\
\hline$c_{r i}$ & $\begin{array}{c}\text { ith group normalized precursor } \\
\text { concentration }\end{array}$ \\
\hline$c_{r}$ & normalized precursor concentration \\
\hline $\mathrm{I}_{\mathrm{r}}$ & normalized iodine concentration \\
\hline $\mathrm{X}_{\mathrm{r}}$ & normalized xenon concentration \\
\hline$T_{f}$ & fuel average temperature \\
\hline$T_{i}$ & $\begin{array}{c}\text { coolant inlet temperature } \\
\text { coolant outlet temperature }\end{array}$ \\
\hline$T_{e}$ & $\begin{array}{c}\text { position variation of the control rod } \\
\text { (fraction of core length) }\end{array}$ \\
\hline$\delta$ rod & $\begin{array}{c}\text { deviation of a parameter from initial } \\
\text { steady-state value }\end{array}$ \\
\hline$\delta$ &
\end{tabular}

One group delayed neutron model is utilized and the coolant inlet temperature is treated as a constant [24].The small perturbation linearization methodology is utilized to linearize the nonlinear core model (1). The transfer function and the state equation of the core are separately calculated and expressed by

$$
\begin{array}{r}
G=\frac{\delta P_{r}}{\delta \operatorname{rod}}=\frac{\sum_{i=0}^{5} a_{i} s^{i}}{\sum_{i=0}^{6} b_{i} s^{i}} \\
\left\{\begin{array}{l}
\dot{\mathbf{x}}=A \mathbf{x}+B u \\
y=C \mathbf{x}+D u
\end{array}\right.
\end{array}
$$

where $u=\delta$ rod-the input; $y=\delta P_{r}$-the output; $a_{i}(i=0,1,2,5)$ numerator coefficients; $\quad b_{i} \quad(i=0,1,2,3,6)$-denominator coefficients; $s$-laplace operator; $\mathbf{x}=\left[x_{1}, x_{2}, x_{3}, x_{4}, x_{5}\right.$, $\left.x_{6}\right]^{\mathrm{T}}=\left[\delta P_{r}, \delta c_{r}, \delta I_{r}, \delta X_{f}, \delta T_{f}, \delta T_{e}\right]^{\mathrm{T}}$-the state matrix; $A$-the $\mathrm{R}^{6 \times 6}$ system matrix; $B$-the $\mathrm{R}^{6 \times 1}$ input matrix; $C$-the $\mathrm{R}^{1 \times 6}$ output matrix; $D$-the zero matrix.

The transfer function $G$ is calculated by using parameters from Ref. [9], in which the total primary heat output is $2200 \mathrm{MW}$, the primary coolant inlet temperature is $285^{\circ} \mathrm{C}$, the primary coolant outlet temperature is $317^{\circ} \mathrm{C}$, the primary coolantaverage pressure is $15.5 \mathrm{MPa}$, and the primary coolant mass flow is $12861.1 \mathrm{~kg} / \mathrm{s}$.

\section{$3 \mathrm{H}_{\infty}$ loop shaping control}

The $\mathrm{H}_{\infty}$ loop shaping design approach was proposed by McFarlane et al. [5] to implement tradeoffs between performance and robust stability of a stable $\mathrm{H}_{\infty}$ loop shaping control system for a plant, where a normalized coprime factor $\mathrm{H}_{\infty}$ robust stabilization issue needs to be solved to guarantee closed-loop stability and a certain level of robust stability of the system at all frequencies.

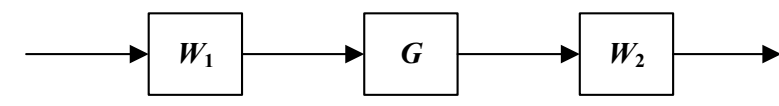

(a)

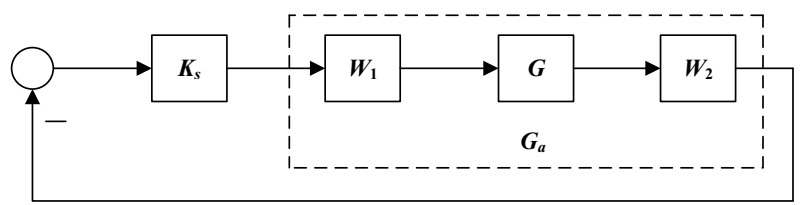

(b)

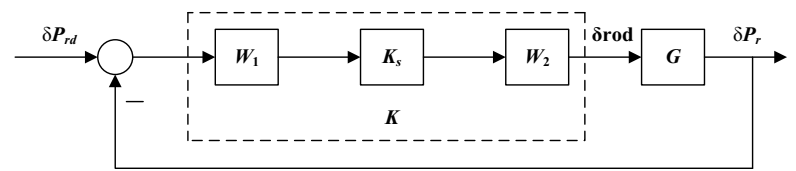

(c)

Figure 1. The loop shaping design procedure for the core.

This $\mathrm{H}_{\infty}$ loop shaping design approach consists of two key techniques, namely loop shape and robust stabilization. The loop shaping design procedure is shown in Fig.1, where $W_{1}$ and $W_{2}$ are weight matrices or compensators; $G_{a}$ is a shaped plant based on $G ; K_{s}$ is the $\mathrm{H}_{\infty}$ controller of $G_{a} ; K$ is the controller to be devised; $\delta P_{r d}$ denotes the reference input, namely the desired change of power level.

(1) Loop shaping

Singular values of a controlled plant $G$ are regulated to obtain a desired open loop shape adopting an additional pre-compensator $W_{1}$ and/or an additional postcompensator $W_{2}$. The plant $G$ is shaped to be $G_{a}$ introducing $W_{1}$ and/or $W_{2}, G_{a}=W_{2} G W_{1}$. Suppose $W_{1}$ and $W_{2}$ are such that $G_{a}$ contains no hidden modes with unstability.

(2) Robust stabilization

Step one: Calculation of $\varepsilon_{\max }$

$\varepsilon_{\max }=\left[\inf _{K \text { stabilizing }}\left\|\left[\begin{array}{l}I \\ K\end{array}\right]\left(I-G_{a} K\right)^{-1} M_{s}^{-1}\right\|_{\infty}\right]^{-1}$

where $M_{s}$ is a normalized coprime factor of $G_{a}$ subjecting to $G_{a}=M_{s}^{-1} N_{s}$.

Step two: Select $\varepsilon \leq \varepsilon_{\max }$, design a stabilizing controller $K_{s}$ satisfying

$$
\left\|\left[\begin{array}{l}
I \\
K_{s}
\end{array}\right]\left(I-G_{a} K_{s}\right)^{-1} M_{s}^{-1}\right\|_{\infty} \leq \varepsilon^{-1}
$$

(3) The eventual feedback controller: $K=W_{1} K_{s} W_{2}$.

The stability margin $\varepsilon$ is both a measurement for robustness of a loop shape control system and the indicator of the success of the loop shaping. Generally speaking, $K_{s}$ is solved so that $\varepsilon \leq \varepsilon_{\text {max }}$ and the controller 
can guarantee that the control system possess satisfied robustness. A small value of $\varepsilon_{\max }$ indicates incompatibility among a desired loop shape, a controlled plant and closed-loop robust stability. In the presence of this case, $W_{1}$ and $W_{2}$ need to be regulated again to obtain a decent loop shape.

\section{Design of nonlinear PWR core power control system}

According to Section 3, solving $K$ is the following.

(1) Loop shaping

$G_{a}$ is exploited and defined as a shaped plant of $G$. Of selected weight matrices for $G, W_{1}$ is $\mathrm{I}_{1 \times 1} ; W_{2}$ is expressed by Eq.(6), and parameters of $W_{2}$ are shown in Table 2, where $\mathrm{e}^{i}=10^{i}$.

$$
W_{2}=\frac{\sum_{i=0}^{6} c_{i} s^{i}}{\sum_{i=0}^{6} d_{i} s^{i}}
$$

Table 2. Parameters of $W_{2}$.

\begin{tabular}{|c|c|}
\hline $\begin{array}{l}\text { Weight } \\
\text { matrix }\end{array}$ & $\begin{array}{l}{\left[c_{6} c_{5} c_{4} c_{3} c_{2} c_{1} c_{0}\right]} \\
{\left[\begin{array}{l}d_{6} \\
d_{5}\end{array} d_{4} d_{3} d_{2} d_{1} d_{0}\right]}\end{array}$ \\
\hline$W_{2}$ & 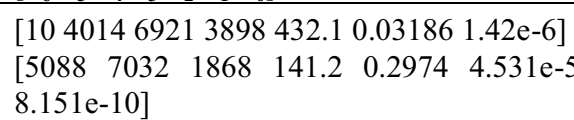 \\
\hline
\end{tabular}

(2) Solve controllers

The controller $K_{s}$ is solved, and then the final controller $K$ of $G$ is calculated by $K=W_{1} K_{s} W_{2} . K$ is expressed by Eq.(7), and parameters of $K$ of $G$ are shown in Table 3 . The solved stability margin $\varepsilon$ of $K$ is shown in Table 3.

$$
K=\frac{\sum_{j=0}^{11} e_{j} s^{j}}{\sum_{j=0}^{12} f_{j} s^{j}}
$$

\begin{tabular}{|c|c|}
\hline Controller & 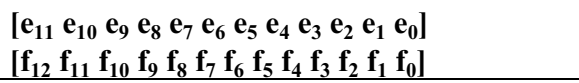 \\
\hline$K$ & $\begin{array}{l}{\left[\begin{array}{lllll}8.06 & 3.625 \mathrm{e} 4 & 1.326 \mathrm{e} 7 & 2.288 \mathrm{e} 7 & 1.291 \mathrm{e} 7 \\
1.452 \mathrm{e} 6 & 2960 & 2.525 & 0.001146 & 2.914 \mathrm{e}-7 \\
3.925 \mathrm{e}-112.184 \mathrm{e}-15\end{array}\right]} \\
{\left[\begin{array}{llllll}1 & 8213 & 1.687 \mathrm{e} 7 & 2.334 \mathrm{e} 7 & 6.233 \mathrm{e} 6 & 4.79 \mathrm{e} 5 \\
1839 & 2.546 & 0.001786 & 7.093 \mathrm{e}-7 & 1.62 \mathrm{e}-10 \\
1.987 \mathrm{e}-14 & 1.015 \mathrm{e}-18\end{array}\right]}\end{array}$ \\
\hline
\end{tabular}

Table 3. Parameters of $K$.

(3) Effect of local controllers

When the reference input $\delta P_{r d}$ is taken as a 0.1 step, responses of the output $\delta P_{r}$ in $G$ with $K$ are shown in Fig.2, where the curve is step response of $\delta P_{r}$ in $G$ with $K$. According to the curve, the output $\delta P_{r}$ in $G$ with $K$ in response to a step possesses good performance, such as the zero overshoot, the short adjustment time and the zero static error. Hence, the controller Kis desirable.

The solved controller $K$ is applied to the nonlinear core model. The combination of the nonlinear core model and $K$ is the nonlinear core power $\mathrm{H}_{\infty}$ loop shapingcontrol system. The schematic of the nonlinear core power $\mathrm{H}_{\infty}$ loop shaping control system is shown in Fig.3.

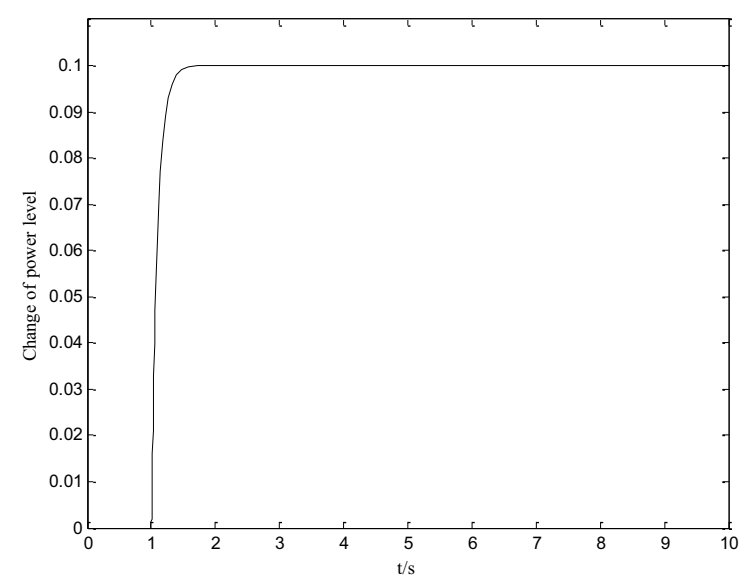

Figure 2. Step response of the output $\delta P_{r}$ from $G_{i}$ with $K_{i}(i=1, \ldots, 7)$.

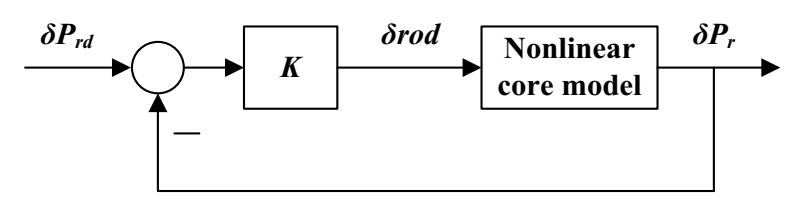

Figure 3. Schematic of the nonlinear core power $\mathrm{H}_{\infty}$ control system.

\section{Simulations}

The nonlinear core power $\mathrm{H}_{\infty}$ loop shaping control systemis simulated considering two typical load variations that are the $-10 \%$ step process and the ramp process with $\pm 0.0556 \% \cdot \mathrm{min}^{-1}$ variations. The direction from the bottom to the top of core is treated as the positive direction of $y$-axis for simulation work, the core bottom is the zero point of $y$-axis and the height of core is $366 \mathrm{~cm}$. When the core power level is at $100 \%$, the initial rod position is at the top of the core, namely at $366 \mathrm{~cm}$.

When the reference input $\delta P_{r d}$ is taken as a $-10 \%$ step load in 24 hours, change trajectories of core parameters during the core following the step load $(100 \% \rightarrow 90 \%)$ are shown in Fig.4, where S1 is the desired load change trajectory for the start of reactorfull power level operation, the middle of reactor shut-down operation with a $-10 \%$ step variation, and the end of $90 \%$ power level operation; $\mathrm{S} 2$ is a change trajectory of the core power level; $\mathrm{S} 3$ is a change trajectory of the control rod position in the core.

When the reference input $\delta P_{r d}$ is taken as a ramp load with $\pm 0.0556 \% \cdot \mathrm{min}^{-1}$ variations, change trajectories of core parameters during the core following the ramp load $(100 \% \rightarrow 90 \% \rightarrow 100 \%)$ are shown in Fig.5, where R1 is the desired load change trajectory for the start of reactor full power level operation, the middle ofreactor shutdown operation with a $-6 \% \cdot \mathrm{min}^{-1}$ ramp variation and $90 \%$ power level operation, and the end of reactor start-up operation with a $6 \% \cdot \mathrm{min}^{-1}$ ramp variation; $\mathrm{R} 2$ is a change 
trajectory of the core power level; R3 is a change trajectory of the control rod position in the core.

Consequently, SL2 (RL2) can follow SL1 (RL1) in real time; meanwhile, the processes of SL3 and RL3 are suitable and acceptable.

Hence, S2 can follow S1, and R2 can follow R1 in real time. Besides, the dynamic processes of $\mathrm{S} 3$ and $\mathrm{R} 3$ are acceptable.
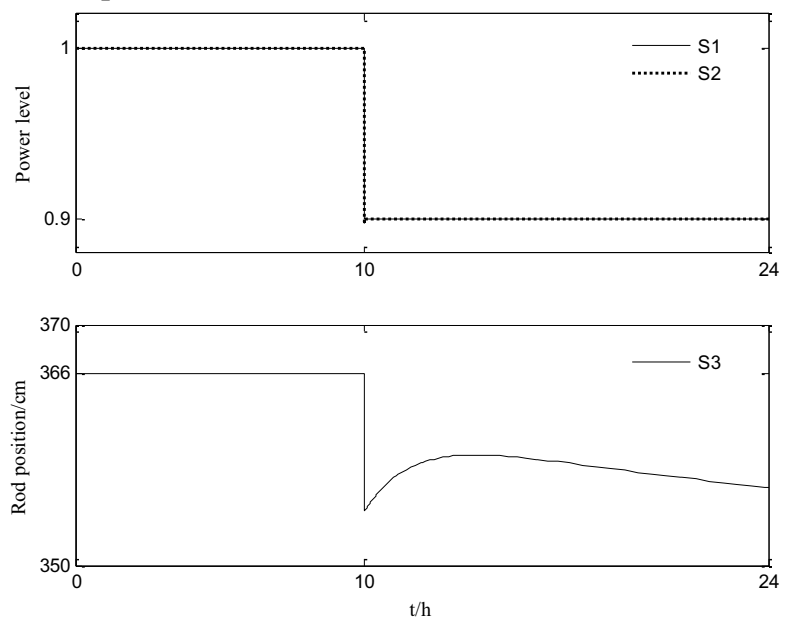

Figure 4. Change trajectories of core parameters during the core following a step load $(100 \% \rightarrow 90 \%)$.
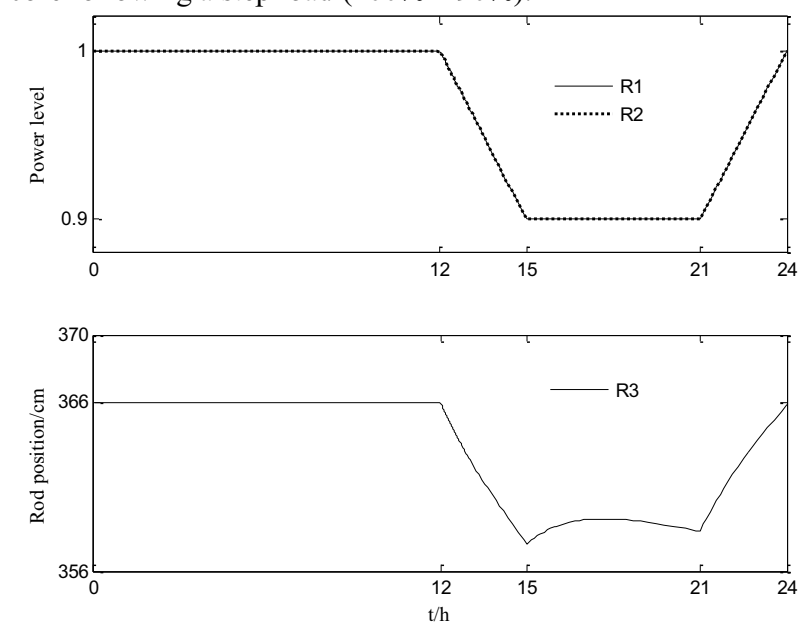

Figure 5. Change trajectories of core parameters during the core following a ramp load $(100 \% \rightarrow 90 \% \rightarrow 100 \%)$.

\section{Conclusions}

The power-level control for PWR cores with xenon ocsillationsis difficult to be implemented, which is also a challenge reaesrch direction. In this paper, the power- level control problem for a PWR core is handled applying the $\mathrm{H}_{\infty}$ loop shapingcontrol strategy to this nonlinear core.

The nonlinear PWR core and its linearized model are separately modeled using the lumped parameter method and the small perturbation linerization method that are capable of be reference modeling approaches to study other nonlinear plants.

In order to implement applications of the controller designed on a linearizedcore model to a nonlinearcore model, the solved $\mathrm{H}_{\infty}$ loop shaping controller has strong robustness of accommodating model errors between the nonlinear model and the linear model.

The devised nonlinear PWR core power $\mathrm{H}_{\infty}$ loop shaping control systemcan control the core powerlevelsatisfactorily in response to different load changes such as a step or ramp. Hence, the nonlinear PWR core power $\mathrm{H}_{\infty}$ loop shaping control system is effective.

\section{Acknowledgement}

The authors would like to thank anonymous reviewers for their valuable comments. The work is funded by National High Technology Research and Development Program of China (863 Program) (No.2015AAXX46611) and China Postdoctoral Science Foundation (No.20159200078).

\section{References}

1. R. M. Edwards, Robust optimal control of nuclear reactors (Pennsylvania State University, 1991)

2. R. M. Edwards, K. Y. Lee, M. A. Schultz, Nuclear Technology, 92, 167-185 (1990).

3. A. Ben-Abdennour, R. M. Edwards, K. Y. Lee, IEEE Trans. on Nuclear Science, 39, 2286-2294 (1992).

4. H. Arab-Alibeik, S. Setayeshi, IEEE Trans. on Nuclear Science, 50, 211-218 (2003).

5. D. McFarlane, K. Glover, IEEE Trans. on Autom. Control, 37, 759-769 (1992).

6. M. A. Schultz, Control of nuclear reactors and power plants (McGraw-Hill, 1961).

7. T. W. Kerlin, E. M. Katz, J. G. Thakkar, J.E. Strange, Nuclear Technology, 30, 299-316 (1976).

8. M. N. Khajavi, M. B. Menhaj, A. A. Suratgar, Annals of Nuclear Energy, 29, 751-760 (2002).

9. IAEA, Directory of Nuclear Reactors Vol.IX, (International Atomic Energy Agency, 1971). 